\title{
Interference characteristics in concurrent discrimination performance by monkeys
}

\author{
F. ROBERT TREICHLER and THOMAS V. PETROS \\ Kent State University, Kent, Ohio 44242
}

\begin{abstract}
Interference effects were evaluated in a series of tests that provided six different conditions of change in objects or reinforcement contingencies within four-problem concurrent tasks. The nine monkeys tested showed little interference relative to a time-only control when novel objects were interposed between acquisition and a retention test. However, if the intervening material provided changes in initial reinforcement contingencies, then substantial retention losses were observed. The results were interpreted as support for the preeminent role of proactive interference in tests of multiple-problem monkey memory.
\end{abstract}

Clarifying the properties of memory shown in animal models has frequently entailed searching for the events that are encoded to support either acquisition or retention (see Rescorla \& Holland, 1982, for review), and a common tactical approach has been to evaluate the consequences of interposing different classes of events between an opportunity for learning and a subsequent memory test. The results of such manipulations have provided a part of the basis for classical interference theory interpretations of memorial processes (Underwood, 1957). Recent studies of interference effects have evaluated the independent or interactive consequences that a series of stimuli may exert upon subsequent choice performance in the delayed matchingto-sample (DMTS) paradigm (Medin, Reynolds, \& Parkinson, 1980). These investigators presented stimuli that bore some relationship to the ones used as trialsetting samples at various times during a retention interval, and they demonstrated that similarities among the physical characteristics or the reward outcomes associated with such stimuli influenced subsequent simultaneous two-choice test performance. Their general conclusion was that either facilitation or interference of memorial processes could be obtained in the DMTS paradigm as a consequence of the retrieval cue properties of interpolated stimuli.

In the present investigations, we have attempted to look at the generality of interference effects by manipulating some qualitative characteristics of task elements when the retention of concurrently learned discriminations is under evaluation. One reason for the choice of this behavior is that prior findings (Treichler, Petros, \& Lesner, 1981) have indicated that concurrent discrimination is not readily subject to retroaction effects

We gratefully acknowledge the assistance of T. A. Mazzaferri in the conduct of this study. Requests for reprints may be sent to either F. R. Treichler, Department of Psychology, Kent State University, Kent, Ohio 44242, or T. V. Petros, Department of Psychology, University of North Dakota, Grand Forks, North Dakota 58202. from intervening new tasks even when relatively large numbers of multidimensional objects have served as discriminanda in both the target and interference tasks. In an effort to treat some issues in common with the Medin et al. (1980) study, the present experiment evaluated the retention of concurrently acquired problems when original learning and interpolated material represented variations of similarity in task elements and/or reward contingencies. One previous study of concurrent discrimination performance (Sledjeski \& French, 1968) is related to the present investigation in its provision of selective changes in task elements and reward contingencies after original concurrent acquisition. While some of the same manipulations were used in the present study and the earlier investigation, the Sledjeski and French (1968) procedures simultaneously imposed six different transfer conditions in association with problems that were presented concurrently, and a question might be raised concerning the independence of treatment effects when so many conditions are changed at one time. In our situation, each treatment condition was associated with only one task and the several individual treatments were imposed in a serial counterbalanced order. Because the major interference effects in Sledjeski and French's (1968) transfer tests were seen when changes in reinforcement contingency were imposed, it was our intention to minimize possible interproblem or general confusion effects by separating the seemingly potent reinforcement change treatments from the potentially more subtle effects of changes in elements of the discriminative stimuli.

\section{METHOD}

Nine adult female rhesus macaques (3.2-5.4 kg) with extensive concurrent discrimination training in the WGTA served as subjects. Soybeans or raisins were used as rewards, common-use objects served as discriminanda, and each animal was administered eight noncorrectional trials in a daily session. Four simultaneous two-choice discrimination problems were presented concurrently, with the same object pairs maintained on a trial, but serial orders of appearance of pairs varied from one 
presentation to the next. Each set of four concurrent problems was designated as a "task," and the criterion for task completion was errorless performance on all trials within a daily session (twice through the four-problem task).

Initial performances entailed training to criterion on one task, then introducing some change in the discriminanda with further training until criterion was again met, and subsequently returning to the original task elements for still another criterion attainment. Thus, the procedures could be characterized as providing acquisition, a retroaction condition, and a retention test for each task. In the first phase of the study, three different tasks were presented as a counterbalanced series and a different retroaction condition was associated with each task. The different conditions imposed after original acquisition were (1) replacement of the incorrect objects in each problem with new objects, (2) replacement of correct objects with new objects, or (3) replacement of both correct and incorrect objects (i.e., a new task). When the entire sequence of tests on all three tasks had been completed, the mean number of days between attainment of the acquisition criterion and initiation of the retention testing was calculated. Thereafter, all subjects were trained to criterion on a fourth task (Task X) and were maintained without any testing for a period equal to the mean time required for administration of retroaction conditions (4 days). At the completion of this period, all subjects were tested for Task X retention by the relearning method.

Phase 2 entailed a test series like that used in the first phase except that different "retroaction" conditions were imposed. In order to evaluate the effects of changes in reinforcement contingencies, three additional tasks and associated conditions were presented. The conditions were (4) reinforcement contingencies were changed for all objects (correct to incorrect and incorrect to correct; i.e., a concurrent reversal), (5) the correct objects became incorrect and new correct objects were provided, and (6) the incorrect objects were designated correct and new incorrects were provided. As in the first phase, the tasks representing various conditions were presented in counterbalanced order and then one more task (Task Y) was provided wherein a period of no testing was interposed between acquisition and retention. The duration of the retention interval ( 9 days) was once again based on the mean number of sessions required to complete the change-treatment segments of this testing phase.

Within each phase of testing, new tasks and conditions for each animal were instituted on the day following criterion attainment on its prior test in the series, and with few exceptions, a schedule of daily test administration was maintained.

\section{RESULTS}

The primary dependent variable in all tests was the number of errors committed before attaining the criterion of errorless performance on the two consecutive daily presentations of a four-problem task. Mean errorsto-criterion scores obtained on the several tasks under various test conditions are displayed in Table 1 .
Tests for homogeneity of variance revealed that violations of this assumption were reflected in some comparisons, particularly within measures of retention. Consequently, both nonparametric and parametric analyses were conducted, but it should be noted that identical patterns in significance of differences were obtained using both kinds of tests. Separate analyses were conducted for the phases of testing that provided changes in only stimulus elements (Phase 1) or in both stimuli and characteristics of reinforcement (Phase 2). Within the first test phase, both Friedman tests and analyses of variance indicated no significant differences $(p<.05)$ among error scores in acquisition or in the scores required to learn the tasks when elements were changed. Similar tests showed no significant differences among retention scores under the various conditions, but $t$ tests and Wilcoxon tests revealed significant (all ps <.02) differences between acquisition and retention scores under all conditions (i.e., there were marked savings). One planned comparison of differential transfer effects was conducted between the conditions in which incorrect or correct discriminanda were replaced (Conditions 1 and 2, respectively). Although acquisition scores between Tasks 1 and 2 did not differ, the errors on the changed version of Task 1 (when only the incorrect objects were replaced) were significantly less than $(\mathrm{t}=2.07, \mathrm{p}<.05)$ those seen on its acquisition. No such difference was observed on Task 2 , in which only the correct objects were replaced.

Analyses of the second phase of testing employed comparisons like those of the first phase, and once again, acquisitions of the four different tasks yielded no significant differences. Further, the error scores were not different from the acquisitions in Phase 1. Comparisons among error scores when the various change conditions were instituted did show significant differences $[F(2,8)$ $\left.=3.47, \mathrm{p}<.05 ; \chi_{\mathrm{r}}^{2}=6.06, \mathrm{p}<.05\right]$, and subsequent Newman-Keuls (and Wilcoxon) comparisons revealed that this significance was based upon differences between outcomes in Conditions 4 and 6. Condition 5 was intermediate and did not differ from either 4 or 6 . Comparisons among retention measures also yielded significant differences $\left[F(3,8)=5.62, p<.01 ; \chi_{\mathrm{r}}^{2}=\right.$ $10.30, \mathrm{p}<.05$ ], with a posteriori testing revealing that these significances were based upon Conditions 4 and 5 both generating greater error than that observed in Condition Y.

Table 1

Mean Errors-to-Criterion Scores for the Various 4-4 Tasks in the Two Phases of the Experiment

\begin{tabular}{|c|c|c|c|c|c|c|c|c|}
\hline & \multicolumn{4}{|c|}{ Phase 1} & \multicolumn{4}{|c|}{ Phase 2} \\
\hline & Task 1 & Task 2 & Task 3 & Task X & Task 4 & Task 5 & Task 6 & Task Y \\
\hline Acquisition & 9.33 & 8.33 & 7.33 & 10.67 & 8.44 & 7.33 & 5.89 & 4.33 \\
\hline Retroaction Condition & $\begin{array}{l}\text { NEW- } \\
4.33\end{array}$ & $\begin{array}{l}\text { NEW+ } \\
7.11\end{array}$ & $\begin{array}{l}\text { NEW+ } \\
\text { NEW- } \\
9.33\end{array}$ & $\begin{array}{l}\text { TIME } \\
\text { ONLY }\end{array}$ & $\begin{array}{l}+ \text { to }- \\
- \text { to }+ \\
21.89\end{array}$ & $\begin{array}{l}+ \text { to }- \\
\text { NEW+ } \\
14.67\end{array}$ & $\begin{array}{l}- \text { to }+ \\
\text { NEW- } \\
10.89\end{array}$ & $\begin{array}{l}\text { TIME } \\
\text { ONLY }\end{array}$ \\
\hline Relearning & .44 & 1.00 & 1.67 & .33 & 16.67 & 9.56 & 8.22 & 1.89 \\
\hline
\end{tabular}

Note-The performance criterion was no error in one daily eight-trial session. 
Pairwise comparisons between the Phase 2 acquisition scores and the scores on the retroaction conditions showed that significantly greater error was associated with the modified tasks for both Conditions 4 and 5 (both ps <.05), but not for Task $6(t=1.36, p>.10)$. Similar comparisons between acquisition and retention measures revealed that significant savings were present only when intervening testing was omitted (Task Y: $\mathrm{t}=3.77, \mathrm{p}<.01)$. Under all change conditions, the animals showed retention error scores that were no different from those required for initial acquisition.

\section{DISCUSSION}

The data in Table 1 and the statistical tests suggest commonalities between the properties of retention in concurrent discrimination and the retention characteristics seen when other behavioral measures of interference are evaluated. In the present first test phase, changes from original discriminanada by replacement with new, novel objects required an amount of training equal to that needed for acquisition, unless the correct objects were maintained. In that case, some transfer to the modified task was present. However, the provision of a set of four new correct objects during interposed training did not impair original task retention any more than that seen either with maintained correct objects or with a control condition wherein no testing occurred.

Second-phase testing entailed acquisitions of four-problem tasks that were like those of the first phase, but here the interposed conditions provided changes in reinforcement contingencies, and these yielded relatively high error scores. Changes in the reward characteristics of both discriminanda or changing the correct objects to incorrect while adding new correct ones yielded significantly elevated error scores. Allowing the previously incorrect object to be designated correct, and adding a new set of incorrect objects also produced some increment in error. However, the amount was not sufficient to produce a reliable difference between the acquisition and retroaction condition scores on Task 6 . Thus, while some negative transfer was derived from changing the incorrect objects to the correct ones, the animals made errors commensurate with learning a new task, and not like the significantly elevated error scores seen on Tasks 4 and 5, on which correct objects changed their reward characteristics. The pattern of outcomes supports previous observations of the relatively greater influence of the correct objects in concurrent tasks and lends support to the characterization of concurrent discrimination as analogous to the learning of a list of correct objects (Treichler, Wetsel, \& Lesner, 1977).

When retention of the original Phase 2 tasks was measured, no savings were evident in any except the control case, in which no testing intervened. Of course, return to the original task also represented a change in reinforcement contingency for
Conditions 4, 5, and 6 , and the outcomes here may be viewed as further evidence of the interference effects associated with manipulations of reward properties.

One property that may be noted in the pattern of results is that impaired retention was not dependent upon the amount of error generated by the intervening change condition. For example, comparison of Conditions 3 and 6 revealed that there were no differences in the error scores associated with either acquisition or intervening tasks. However, retention was significantly less proficient when reinforcement contingencies were manipulated, and this outcome seemed attributable, not to the amount of error on the intervening task, but to the nature of interfering conditions.

In overview, the results from this experiment do show parallels with the Medin et al. (1980) study and suggest that interference has predictable influences in tests other than those of short-term memory. Within our concurrent paradigm, as in their DMTS test, various interfering stimuli can produce enhancement, impairment, or relatively little effect upon retention. Further, because impairment in the present tests is restricted to conditions that involve changes from prior reinforcement contingencies, it appears that, although the monkey's retention of many concurrently learned problems is excellent, this facility serves to impair performance when the target tests require overcoming elements of proaction from retained information. Indeed, the characterization of primate memory that seems most warranted on the basis of several concurrent discrimination results is that it may be described as relatively little influenced by retroaction but much affected by proactive interference.

\section{REFERENCES}

Medin, D. L., Reynolds, T. J., \& Parkinson, J. K. Stimulus similarity and retroactive interference and facilitation in monkey short-term memory. Journal of Experimental Psychology: Animal Behavior Processes, 1980, 6, 112-125.

Rescorla, R. A., \& Holland, P. A. Behavioral studies of associative learning in animals. Annual Review of Psychology, 1982, 33, 265-308.

Sledjeski, M., \& French, G. M. Multiple transfer paradigms following discrimination problem solution in monkeys. Journal of Comparative and Physiological Psychology, 1968, 66, 228231.

Treichler, F. R., Petros, T. V., \& Lesner, S. A. Preference effects on acquisition and retention of concurrent discriminations by rhesus monkeys. Animal Learning \& Behavior, 1981, 9, $401-405$.

Treichler, F. R., Wetsel, W. C., \& Lesner, S. A. Some characteristics of concurrent discrimination and retention by monkeys. Learning and Motivation, 1977, 8, 213-228.

UNDERWOOD, B. J. Interference and forgetting. Psychological Review, 1957, 64, 49-60.

(Received for publication January 31, 1983.) 